\title{
Scintillator Evaluation for High-Energy X-Ray Diagnostics
}

September 2001 


\section{DISCLAIMER}

This report was prepared as an account of work sponsored by an agency of the United States Government. Neither the United States Government nor any agency thereof, nor any of their employees, makes any warranty, expressed or implied, or assumes any legal liability or responsibility for the accuracy, completeness, or usefulness of any information, apparatus, product, or process disclosed, or represents that its use would not infringe privately-owned rights. Reference herein to any specific commercial product, process, or service by trade name, trademark, manufacturer, or otherwise, does not necessarily constitute or imply its endorsement, recommendation, or favoring by the United States Government or any agency thereof. The views and opinions of authors expressed herein do not necessarily state or reflect those of the United States Government or any agency thereof.

Available to U.S. Department of Energy and its contractors in paper from:

U.S. Department of Energy

Office of Scientific and Technical Information

P.O. Box 62

Oak Ridge, TN 37831-0062

(423) 576-8401

Available to the public from:

U.S. Department of Commerce

National Technical Information Service

5285 Port Royal Road

Springfield, VA 22161

(703) $487-4650$

Available electronically at http://www.doe.gov/bridge. Available to U.S. Department of Energy and its contractors in paper from:

U.S. Department of Energy

Office of Scientific and Technical Information

P.O. Box 62

Oak Ridge, TN 37831-0062

(423) 576-8401 


\section{Scintillator Evaluation for High-Energy X-Ray Diagnostics}

September 2001

Stephen S. Lutz

Stuart A. Baker

This work was supported by the U.S. Department of Energy, National Nuclear Security Administration Nevada Operations Office, under Contract No. DE-AC08-96NV11718. 


\section{CONTENTS}

$1.0 \quad$ SUMMARY

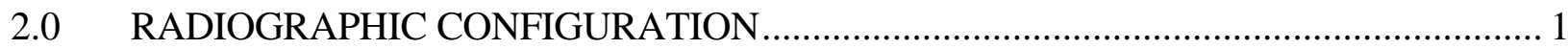

2.1 Detection Apparatus and Measurements........................................................... 2

3.0 LSO THICKNESS OPTIMIZATION …………….............................................. 3

3.1 LSO DQE(0) Calculations .............................................................................. 3

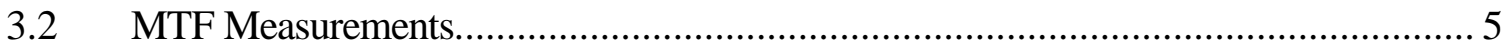

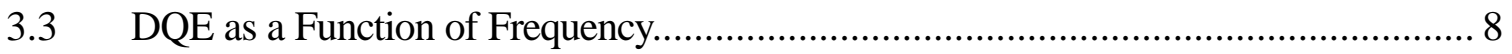

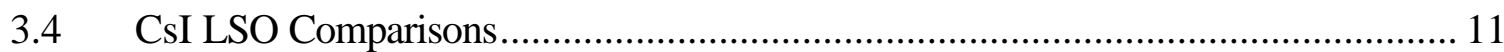

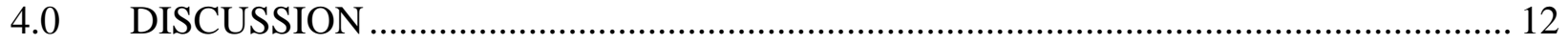

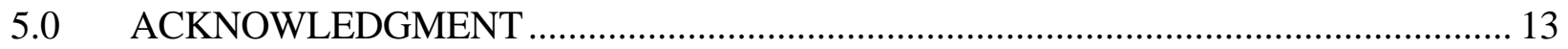

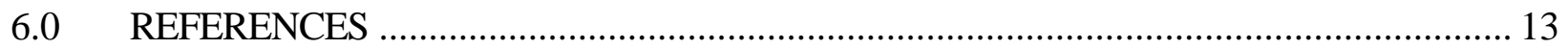




\subsection{SUMMARY}

This report presents results derived from a digital radiography study performed using x-rays from a 2.3 $\mathrm{MeV}$, rod-pinch diode. Detailed is a parameter study of cerium-doped lutetium ortho-silicate (LSO) scintillator thickness, as it relates to system resolution and detection quantum efficiency (DQE). Additionally, the detection statistics of LSO were compared with that of CsI(TI). As a result of this study we found the LSO scintillator with a thickness of $3 \mathrm{~mm}$ to yield the highest system DQE over the range of spatial frequencies from 0.75 to $2.5 \mathrm{~mm}^{-1}$.

\subsection{RADIOGRAPHIC CONFIGURATION}

This study was performed using a variant of the SABRE pulse-power source at Sandia National Laboratories (SNL). The accelerator ( $2.3 \mathrm{MeV}, 40 \Omega, 60 \mathrm{~ns}$ ) was fitted with a rod-pinch diode to create a high-brightness $\mathrm{x}$-ray source. The anode was a 0.5 -mm-diameter gold rod. This configuration is capable of a $0.85 \mathrm{~mm}$ x-ray spot and a filtered dose of 3.5 rads at $1 \mathrm{~m} .{ }^{1}$ The experimental setup is schematically shown in Figure 1. The SABRE x-ray source is collimated with a first conjugate distance of $1.0 \mathrm{~m}$. The beam is then attenuated by a test object, and the resulting image detected by a scintillator. The target to scintillator distance was approximately $30 \mathrm{~cm}$, resulting in a radiographic magnification of 1.3 .

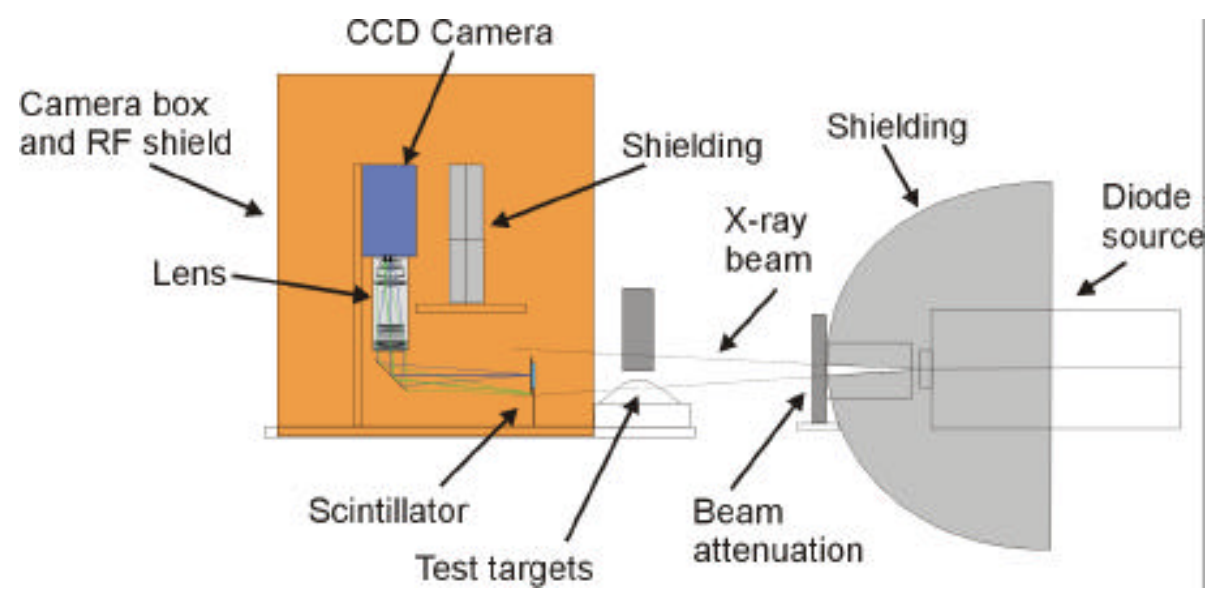

Figure 1. Set up for SABRE x-ray experiments.

The scintillator images are recorded with a CCD camera imaging system. The CCD camera and scintillator are enclosed in a copper screen box, which is electrically isolated to protect the sensitive electronics from electro-magnetic pulse noise generated when SABRE is fired. A lead radiationshielding dome is located around the x-ray source to reduce scattered radiation. The x-ray beam diameter is set by a collimator, which is positioned in the exit port of the shielding dome. Beam attenuation is placed at the output of the collimator as needed to control signal levels and harden the $\mathrm{x}$ ray spectra. Additional radiation shielding is placed inside the screen box to minimize noise in the image generated by radiation scattered onto the CCD camera. Camera control and image acquisition are accomplished through a fiber optic link connecting the CCD camera to a data acquisition computer located in a remote screen room. 


\subsection{Detection Apparatus and Measurements}

These measurements were performed using an optical camera system similar to one designed for DARHT applications by LANL DX-3. The camera, made by Princeton Instruments, has a 2048 x 2048 pixel back-thinned SITe CCD focal plane and is cooled with liquid nitrogen. The system uses a fast $\mathrm{f} \# 1.4$, focal length $=180 \mathrm{~mm}$ custom lens manufactured by Linos. The magnification of this fixed conjugate optical relay is 0.33 . The $24 \mu \mathrm{m}$ CCD pixel dimension, combined with the 0.33 optical magnification, produces a scintillator plane optical sampling period of $72.7 \mu \mathrm{m}$. The Linos lens is specially designed for low distortion and transmission in the blue to accommodate the 400 -to- $500 \mathrm{~nm}$ scintillator emission. The lens views the scintillator through a turning mirror to move the camera and lens out of the primary radiation beam path.

This study characterized several different scintillator parameters in an attempt to determine an optimum scintillator configuration. LSO samples 0.5-, 0.95-, 3.0-, 5.6-, and 7.75-mm-thick were evaluated. The LSO samples were $60 \mathrm{~mm}$ in diameter, mounted in a free-standing holder with edges masked and both flat surfaces polished and anti-reflection coated. Performance parameters investigated include scintillator limiting resolution and modulation transfer function. Additionally, signal-to-noise characteristics were calculated for use in dynamic range, DQE, and noise power spectrum (NPS) measurements. Finally, a signal-to-noise ratio (SNR) comparison was performed between a 2.2-mmthick LSO array and a 2.2-mm-thick monolithic sample of CsI:Tl.

Figure 2 shows typical imagery from three different thickness scintillators. Shown in the radiographs are a series of bar targets arranged along a partially opaque edge. The bar targets are constructed of a series of Ta shims, 10-mm-thick in the radiographic axis. The edge consists of a 3-mm-thick Ta block. The spatial frequencies of the bar targets are 0.51, 1.0, 2.1, 3.2, and $5.3 \mathrm{~mm}$. On the right side of the edge is a $\mathrm{Pb}$ stepwedge. The stepwedge is constructed with 2-mm wide steps, ranging in thickness from 0.9 to $53 \mathrm{~mm}^{-1}$. The SNR of c) appears poor because $49 \mathrm{~mm}$ of steel was added to the beam to prevent saturation of the camera system. The x-ray transmission of the steel is approximately $5 \%$.

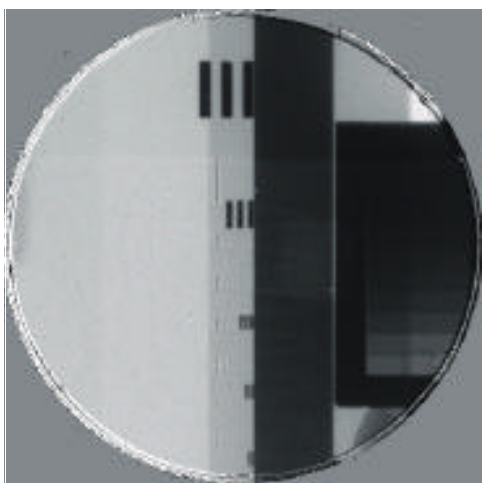

a)

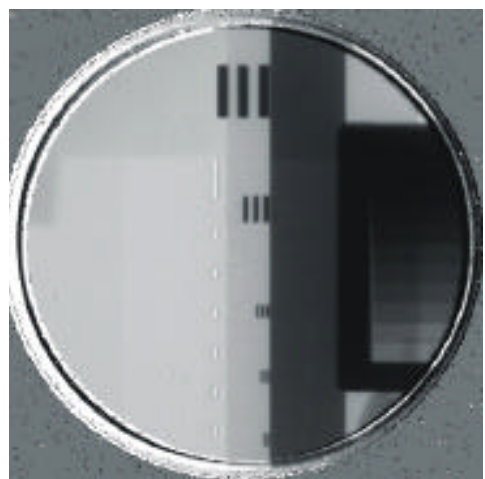

b)

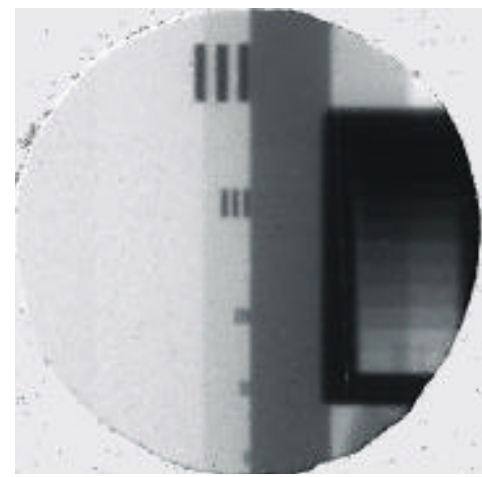

C)

Figure 2. SABRE images as a function of LSO thickness for a) 0.5 , b) 3.0 , and c) $7.75 \mathrm{~mm}$. 


\subsection{LSO THICKNESS OPTIMIZATION}

For a given x-ray energy spectrum, digital radiographic system performance is a strong function of scintillator thickness. Designing a system for which spatial resolution is the driving concern favors a relatively thin converter. Primary sources of image blur include radiation scatter in the scintillator and depth-of-focus of the optical system. Both of these blur sources are functions of scintillator thickness. Conversely, designing a system in which statistical detection is of paramount importance favors a thicker converter. X-ray absorption statistics and visible light production both increase with scintillator thickness. These competing effects lead to a relatively narrow optimal range of scintillator thickness for a specified x-ray energy. To select the optimum scintillator thickness, DQE as a function of spatial frequency is used as a figure-of-merit.

$\mathrm{DQE}$ can be expressed as the ratio of the square of the SNR of a real detection system to that of a perfect, quantum limited detector.

$$
D Q E=\frac{S N R_{\text {out }}{ }^{2}}{S N R_{\text {in }}{ }^{2}}
$$

The approach of this optimization is to calculate zero-frequency DQE data, based upon x-ray source spectra, NIST absorption tables, published scintillator data, and camera configuration information. This zero-frequency DQE calculation is then combined with experimental results, detailing resolution and NPS of the detection system. The result is a three-dimensional image relating DQE, frequency, and scintillator thickness.

\subsection{LSO DQE(0) Calculations}

As described in Watson's report ${ }^{2}$, for a mono-energetic gamma beam, the zero-frequency value of the $\mathrm{x}$-ray $\mathrm{DQE}(0)$ can be calculated by:

$$
\operatorname{DQE}(0)=\frac{1-\mathrm{e}^{-\mu \mathrm{t}}}{1+\frac{1}{\mathrm{n}}}=\frac{\mathrm{ABS}_{\text {scintillator }}}{1+\frac{1}{\mathrm{n}}}
$$

Where $n$ is the mean number of recorded visible light quanta per gamma ray quanta absorbed, and $\mu$ is the absorption coefficient for the $\mathrm{x}$-ray photon in the scintillator material. The system conversion efficiency $(n)$ can be calculated by the following:

$$
n=\frac{Q E_{C C D} * G^{*} E_{e f f} * M^{2}}{16^{*} f_{\#}^{2} * \eta^{2} *(M+1)^{2}}
$$

For a non-mono-energetic gamma source, the scintillator absorption $\left(1-e^{-\mu t}\right)$, is energy dependent and must be calculated as a weighted sum. That is, the sum of the product of the source spectra times the scintillator absorption, divided by the total incident flux:

$$
\mathrm{ABS}_{\text {scintillator }}=\frac{\sum_{\mathrm{E}} \text { Flux }(\mathrm{E}) *\left(1-\mathrm{e}^{-\mu(\mathrm{E}) \mathrm{t}}\right)}{\sum_{\mathrm{E}} \operatorname{Flux}(\mathrm{E})}
$$


The $\mathrm{G}$ value of the scintillator (Eq. 3), in units of photons/MeV, is also energy dependant. However, for a scintillator thickness that is "thin" with respect to the incident $\mathrm{x}$-ray spectra in the hundreds of keV range, this variation is assumed to be small. Using this assumption, the $\mathrm{G}$ value was multiplied by the mono-energetic equivalent energy of the gamma beam $\left(\mathrm{E}_{\text {eff }}\right)$ to obtain an average scintillation photon yield per x-ray quanta absorbed. This effective energy is defined as the value that results in a scintillator transmittance equal to that calculated using the SABRE source spectra transmitted through the experimental configuration (targets, windows etc).

It should be noted that the $\mathrm{DQE}(0)$ is a function of the incident $\mathrm{x}$-ray spectra, and therefore varies with the amount of material in the $\mathrm{X}$-ray beam. As the effective energy of the beam rises, the scintillator become more transparent to the incident $x$-rays, and the calculated $\mathrm{DQE}(0)$ drops.

Figure 3 shows the results of two calculations of DQE(0) versus LSO:Ce scintillator thickness over a scintillator thickness range of 0.5 to $7.5 \mathrm{~mm}$. The first calculation has $25 \mathrm{~mm}$ of $\mathrm{Al}$ in the beam. The second calculation has an additional 10-mm thickness of $\mathrm{W}$ added to the Al. The parameters in Table 1 describe the scintillator and optical detection system for these calculations.

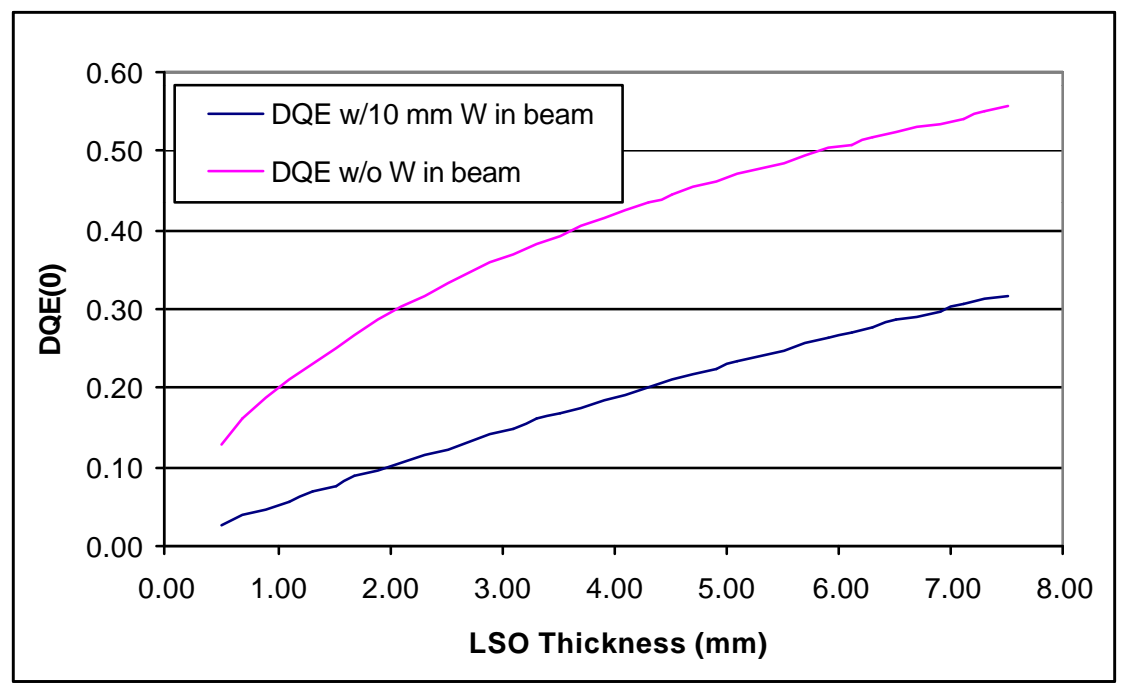

Figure 3. $\mathrm{DQE}(0)$ as a function of scintillator.

Table 1. Scintillator and detection system parameters.

\begin{tabular}{|l|c|l|}
\hline $\mathrm{G}$ & 30000 & literature value $^{3}$ \\
\hline$\mu$ & & energy absorption cross section from NIST XCOM data base $^{4}$ \\
\hline Flux(x) & & Sandia calculated $2.3 \mathrm{MeV}$ endpoint rod-pinch diode spectra \\
\hline QEccd & 0.5 & quantum efficiency of CCD \\
\hline $\mathrm{M}$ & 0.33 & system optical magnification \\
\hline $\mathrm{c}$ & 1.82 & optical index of scintillator \\
\hline $\mathrm{f} \#$ & 1.4 & infinite conjugate lens f\# \\
\hline
\end{tabular}


The addition of the Tungsten raises the effective energy of the beam. When the effective energy is calculated using a $3 \mathrm{~mm}$ LSO scintillator, it increases from $408 \mathrm{keV}$ to $862 \mathrm{keV}$ with the addition of the Tungsten. As shown in Figure 3, DQE drops over a factor of two as the beam is "hardened".

The lower DQE curve, reflecting the "hardened" x-ray spectra, is used for the calculated results in later sections.

\subsection{MTF Measurements}

The detection system modulation transfer function (MTF) was measured for scintillators ranging in thickness from 0.5 to $7.75 \mathrm{~mm}$. The MTF was derived from the Fourier transform of a line-spread function. The line-spread function was obtained by digital differentiation of a radiographic step response created by placing a Ta edge in the radiographic object plane.

Figures 4 a) to 4 e) show a composite set of these measurements. The MTF functions show an expected trend of continual degradation of resolution with increasing scintillator thickness. MTF is seen to degrade across the entire frequency band as scintillator thickness is increased. While these measurements represent a convolution of the entire system, including beam spot, scintillator, and camera response, the magnitude of the change of these MTF functions shows that scintillator thickness is a dominant term at $\mathrm{T}>0.5 \mathrm{~mm}$. As scintillator thickness increases, the power spectra are truncated at ever decreasing frequencies. This was done to constrain the influence of the noise on the fit parameters when the noise becomes a sizeable fraction of the signal.

Each of these MTF spectra were fit using a sum of three, zero-frequency-centered Gaussian functions. This can be written as follows:

$$
\text { MTF }=a^{*} e^{-\frac{1}{2}\left(\frac{v}{b}\right)^{2}}+c * e^{-\frac{1}{2}\left(\frac{v}{d}\right)^{2}}+e^{*} e^{-\frac{1}{2}\left(\frac{v}{f}\right)^{2}}
$$

where $\tilde{o}$ is the frequency in units of $\mathrm{mm}^{-1}$, the parameters a, c, e represent the Gaussian amplitudes, and the parameters b, d, f are the Gaussian widths in frequency units. The use of a three-Gaussian function fit resulted in a substantial improvement in the fit quality versus a two-Gaussian function. The fits are shown as the solid lines in Figures 4 a) to 4 e). The fit parameters are given in the header of each plot.

These Gaussian parameters were then fit to arbitrary analytic functions in order to obtain a continuous smooth function, describing the relationship between system MTF and scintillator thickness. These parameter fits are shown in Figure 5 a) to $5 \mathrm{f}$ ). The result of this parameterization is a smooth threedimensional function that relates MTF, spatial frequency, and scintillator thickness. This function is plotted in image format in Figure 6. In this figure, the x-axis is scintillator thickness in units of mm, the yaxis spatial frequency in units of $\mathrm{mm}^{-1}$, and the z-axis is color encoded with a banded rainbow showing the value of the MTF. 


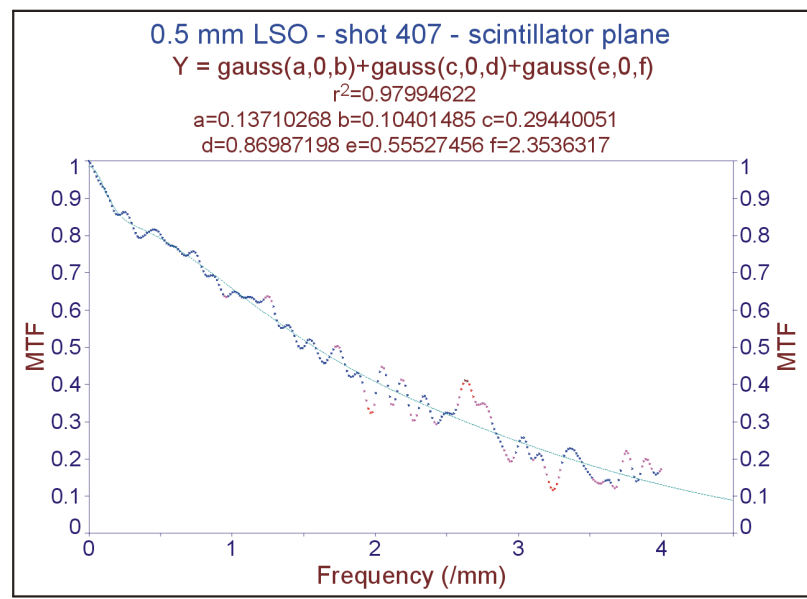

a)

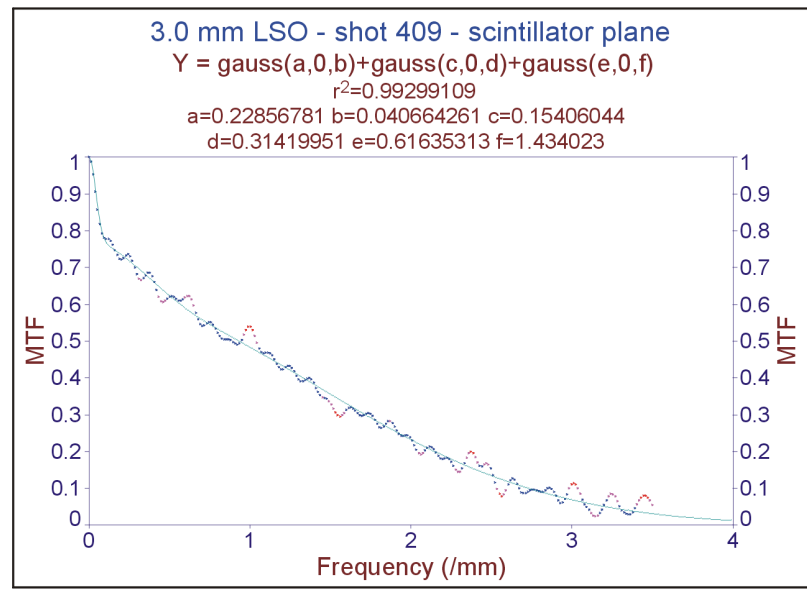

c)

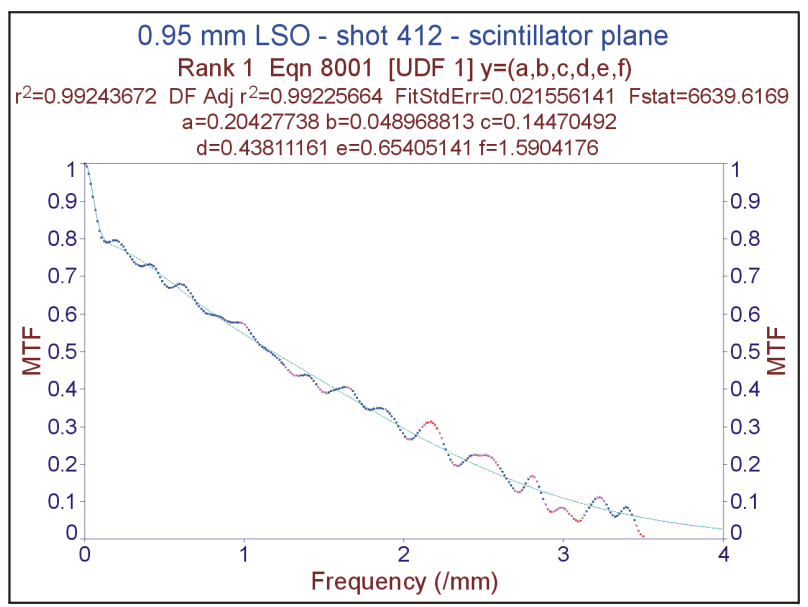

b)

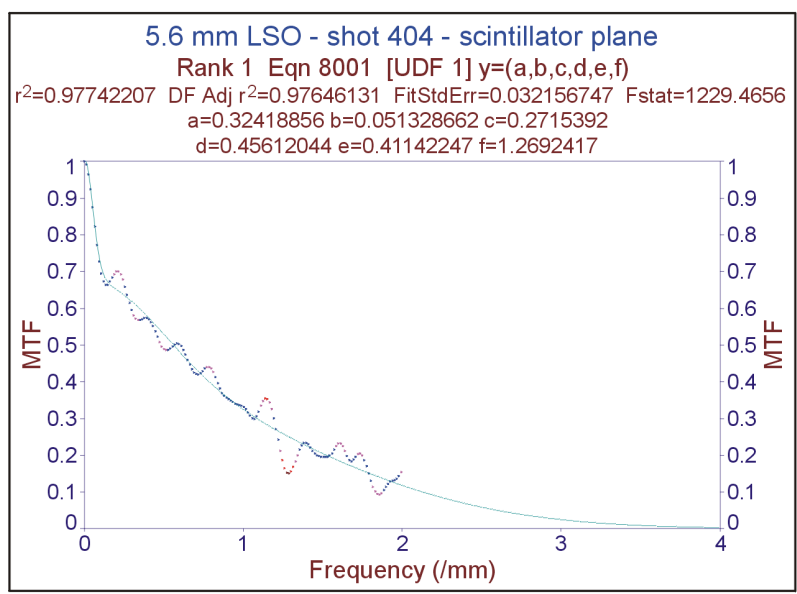

d)

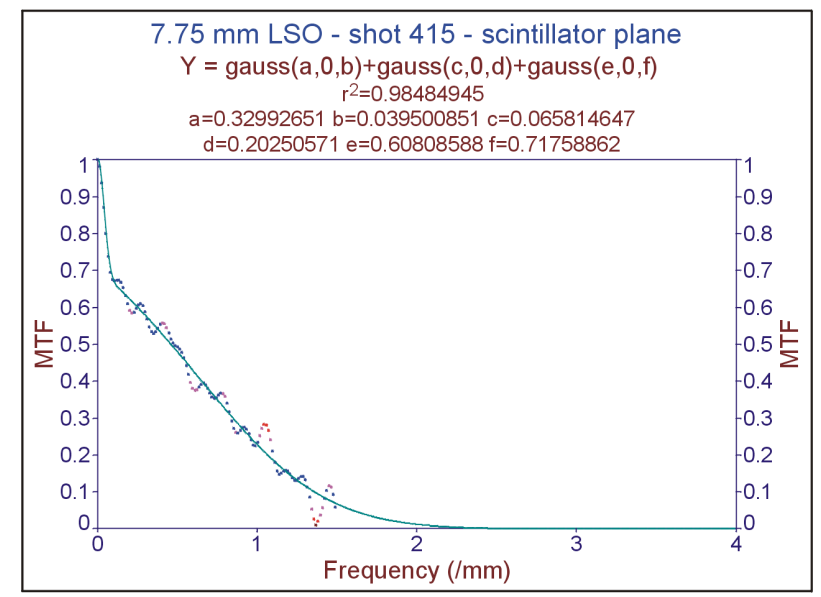

e)

Figure 4. MTF measurements with Gaussian fit. 


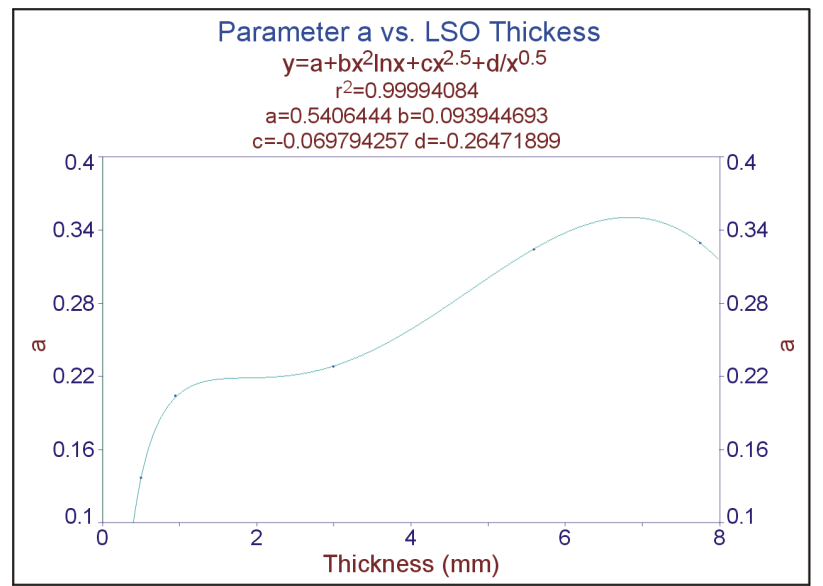

a)

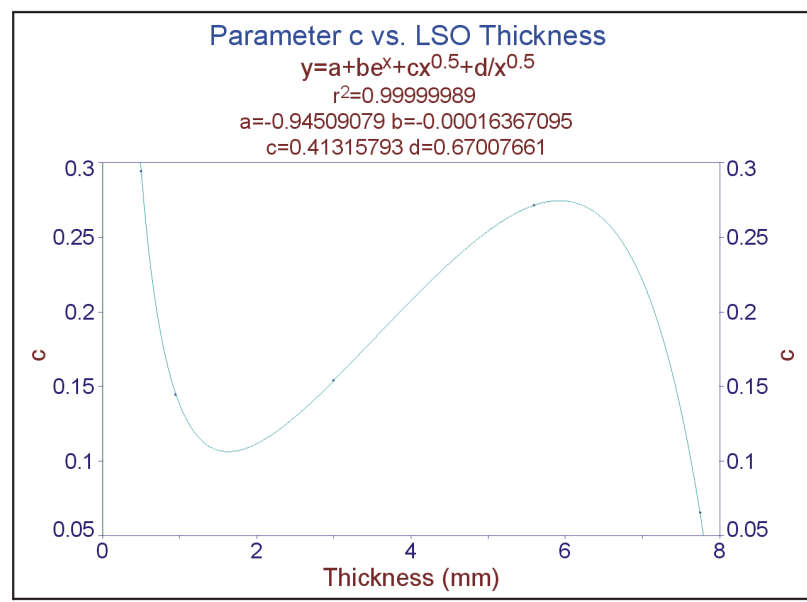

C)

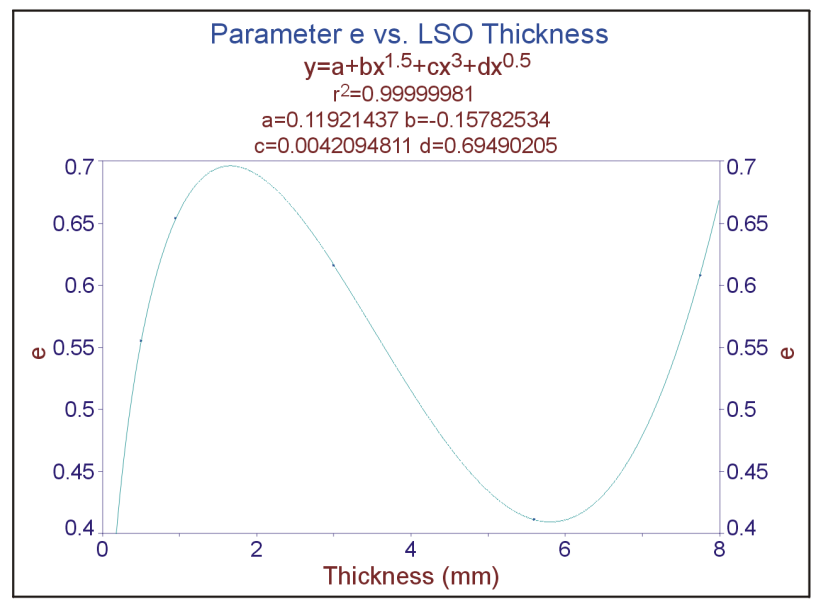

e)

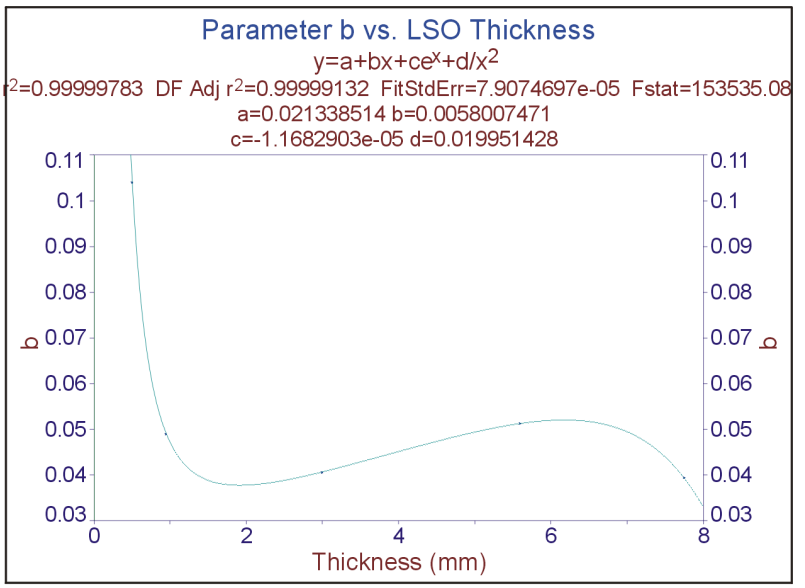

b)

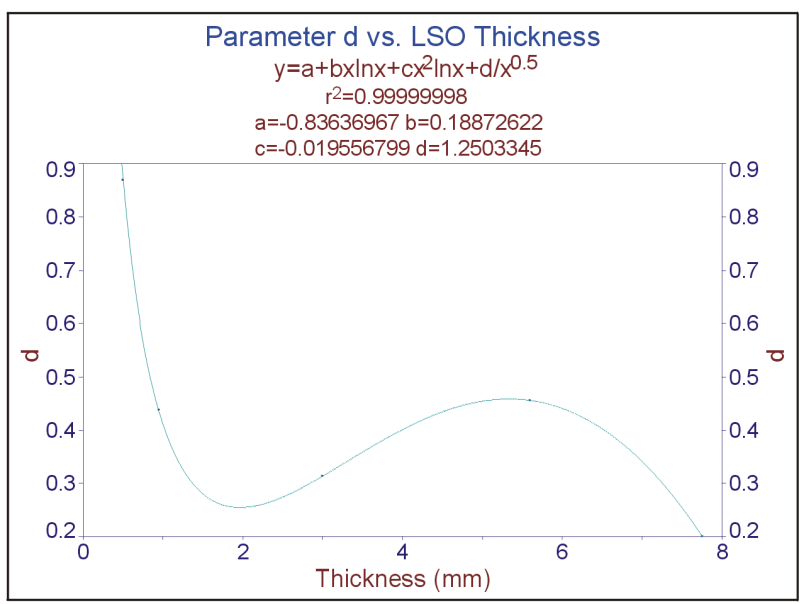

d)

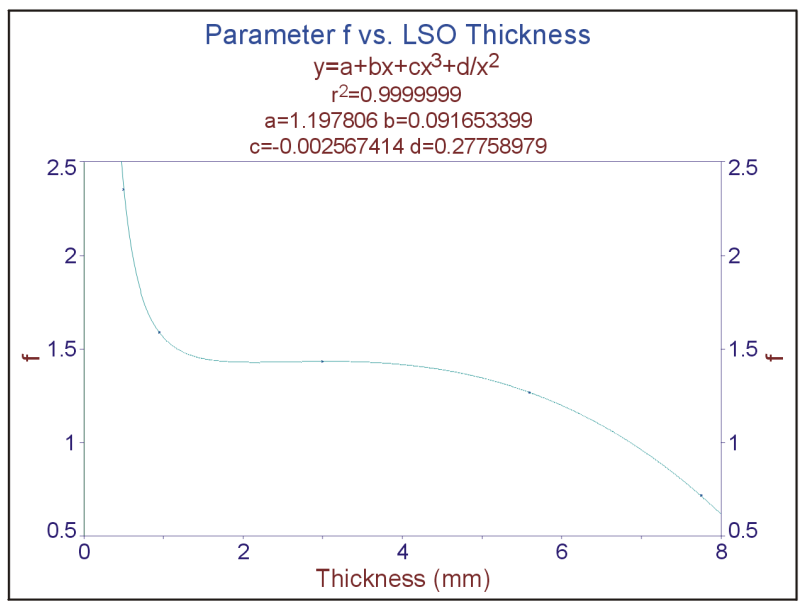

f)

Figure 5. Gaussian parameters fit as a function of thickness. 
The banding of the rainbow allows one to see contours in the three-dimensional data. The image presentation allows one to determine MTF for any thickness scintillator by taking a vertical line slice from the $\mathrm{x}-\mathrm{y}$ plane.

\subsection{DQE as a Function of Frequency}

Dainty and Shaw define the frequency dependence of DQE as a product of the zero frequency value of the DQE and the MTF squared divided by the normalized NPS. ${ }^{5}$ Adding a second dimension, that of scintillator thickness $(\mathrm{t})$, this formula becomes:

$$
D Q E(v, t)=D Q E(0, t) * \frac{M T F(v, t)^{2}}{\operatorname{NPS}(v, t)}
$$

$\operatorname{DQE}(0, t)$ is shown graphically in Figure 3 and $\operatorname{MTF}(v, t)$ is shown in Figure 6 . The NPS $(v, t)$ is the normalized noise power spectrum.

The NPS is derived from the Fourier transform of the auto-correlation of the noise. A noise field was obtained by subtracting two radiographic images, and defining a region-ofinterest in a featureless area of the image. Figure 7 shows the spatial domain results of auto-correlation calculations, in units of pixels versus normalized auto-correlation coefficient, for each LSO thickness. No apparent trend as a function of thickness was discerned. 'Wings' in the auto-correlation function are clearly obvious in the $0.95 \mathrm{~mm}$ data. The shape of the $3 \mathrm{~mm}$ data is also different from the rest. Because no trend as a function of thickness was found, all these data were averaged, transformed and fit to an analytic function. This analytic function, independent of thickness, was then used for the calculation of $\mathrm{DQE}(v, t)$. The results of this DQE calculation are shown in Figure 8.

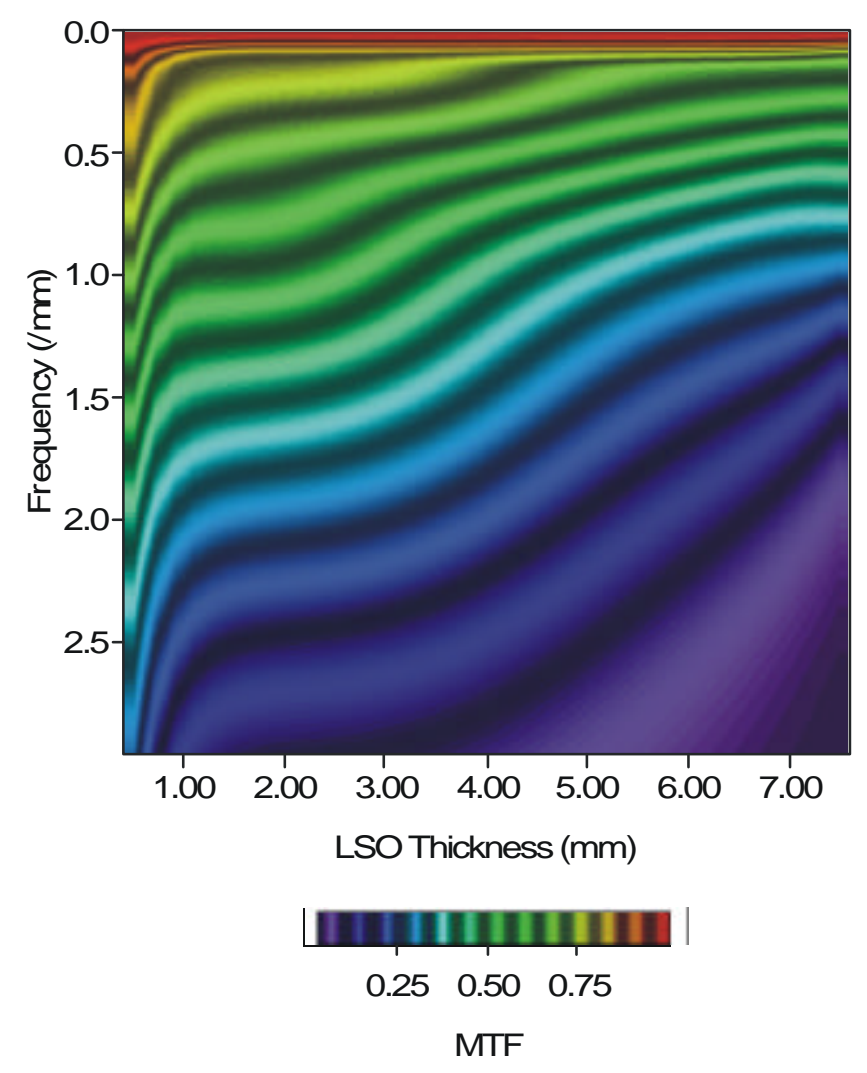

Figure 6. MTF as a function of spatial frequency and scintillator thickness. 


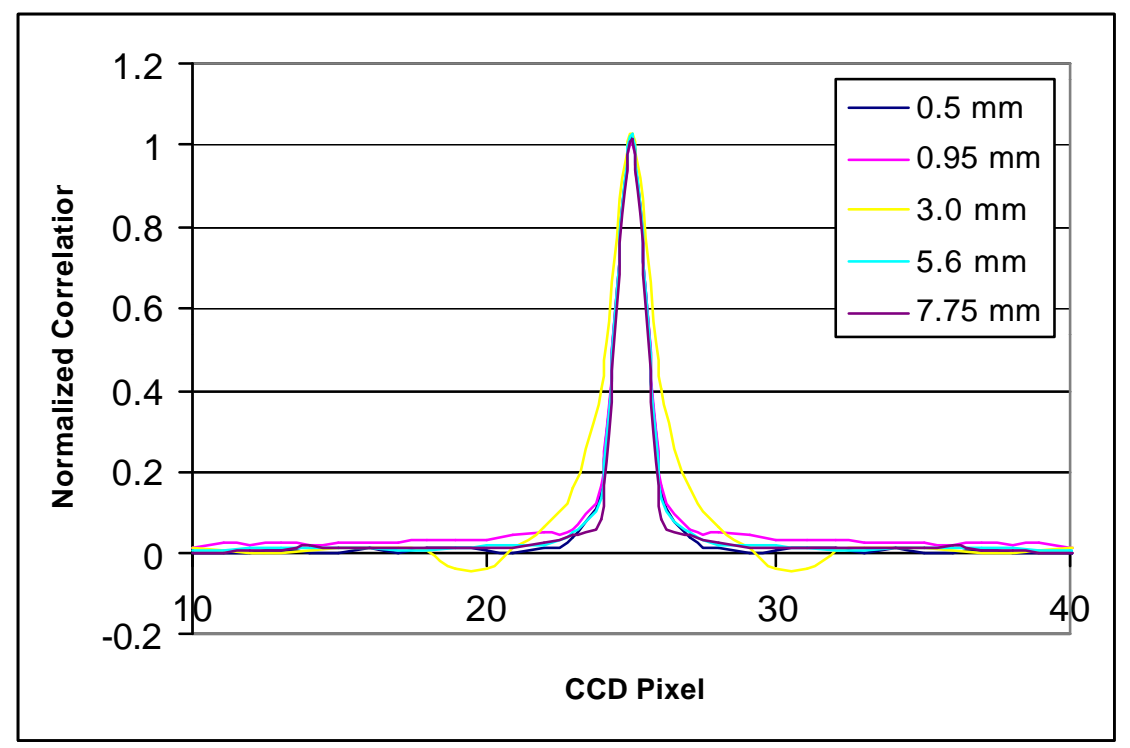

Figure 7. Auto correlation function for different scintillator thickness.

As seen in Figure 8, system performance, vis-àvis DQE, is peaked at a specific scintillator thickness. Further, the location of the peak is dependent on spatial frequency. Plotted semilogarithmically in Figure 9 are four horizontal line slices taken from Figure 8 at spatial frequencies of $0,0.5,1.0,2.0$, and $3.0 \mathrm{~mm}^{-1}$. At low spatial frequencies $\left(<0.5 \mathrm{~mm}^{-1}\right)$, the DQE is peaked at a thickness outside the range of this calculation $(>8$ $\mathrm{mm})$. As the spatial frequency is increased to between 1 and $2 \mathrm{~mm}^{-1}$, the peak in performance for this system occurs at a scintillator thickness of approximately $3 \mathrm{~mm}$. Between 2 and $3 \mathrm{~mm}^{-1}$, the actual peak in system performance shifts back to a thin, $0.5 \mathrm{~mm}$ scintillator. The apparently small increase in the DQE at $3 \mathrm{~mm}^{-1}$ between scintillator thickness of 1.3 and $3 \mathrm{~mm}$ represents a change of nearly $50 \%$.

Figure 10 shows a set of six vertical line slices taken from Figure 8 at scintillator thickness of $0.5,1.1,2.1,3.1,4.1 \mathrm{~mm}$, and $5.1 \mathrm{~mm}$. At spatial frequencies less that

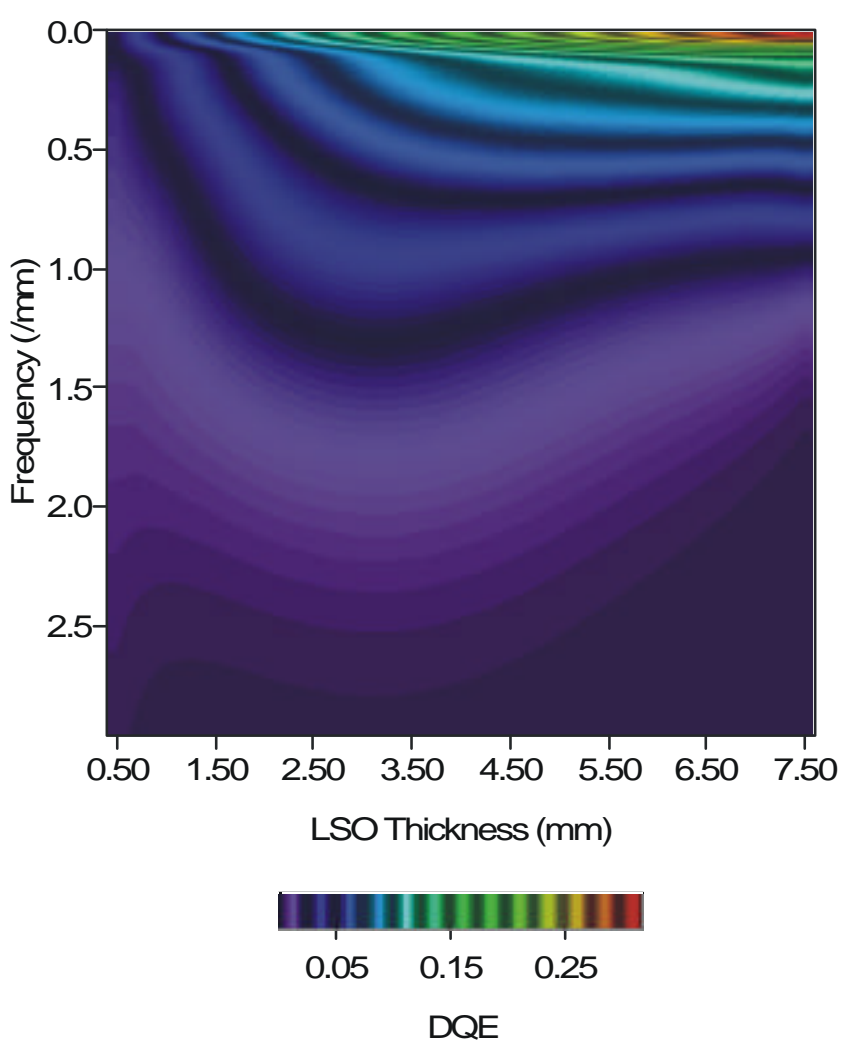

Figure 8. DQE as a function of frequency and scintillator thickness. 
$2.5 \mathrm{~mm}^{-1}$, DQE continually improves with increasing scintillator thickness up to $3.1 \mathrm{~mm}$. Above that thickness, DQE continues to improve at very low $\left(<0.75 \mathrm{~mm}^{-1}\right)$ spatial frequencies, however performance at higher frequencies is degraded. The superior performance of the $0.5 \mathrm{~mm}$ scintillator at frequencies $>2.5 \mathrm{~mm}^{-1}$ can also be seen in this graph.

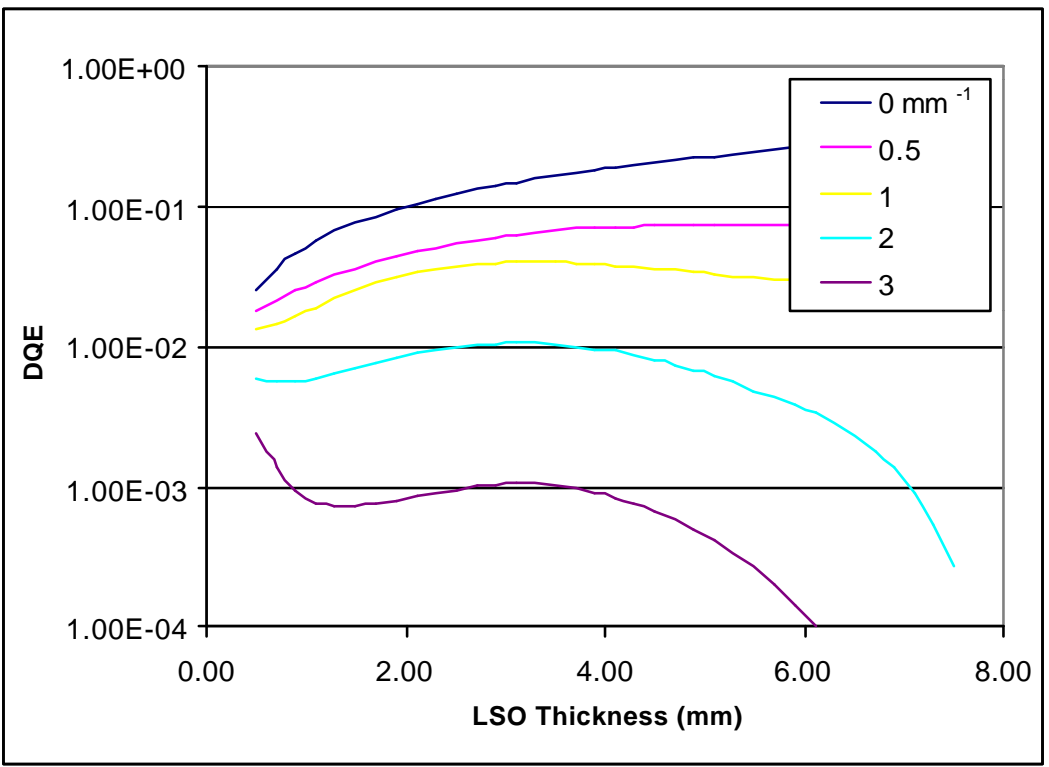

Figure 9. DQE as a function of thickness for five different spatial frequencies.

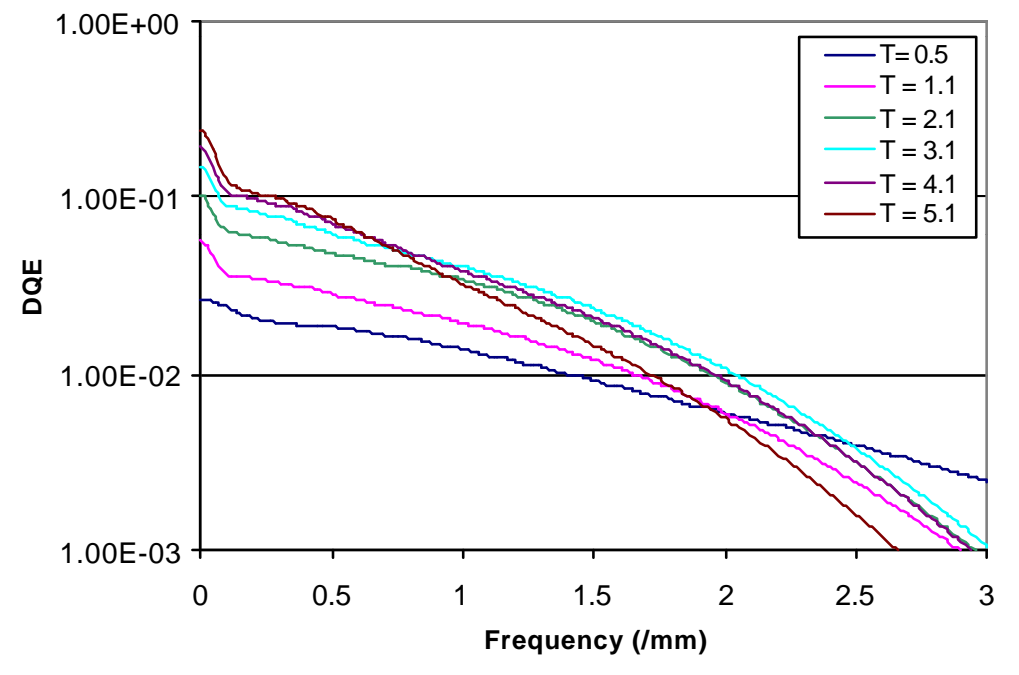

Figure 10. DQE as a function of spatial frequency for six different scintillator thicknesses. 


\subsection{CsI LSO Comparisons}

Cesium Iodide (CsI) is a potential alternative to LSO as a converter material for digital radiography. Advantages of CsI include availability in relatively large $(150 \times 150 \mathrm{~mm})$ monolithic pieces, green emission when doped with Thallium, and a $\mathrm{G}$ value (photons/MeV) about twice that of LSO. The disadvantages of CsI, relative to LSO, are a lower average Z, with a resultant lowered gamma cross section, as well as much lower density $\left(4.51\right.$ versus $\left.7.4 \mathrm{~g} / \mathrm{cm}^{3}\right)$.

These competing parameters ( $\mathrm{G}$ value and density and wavelength/CCD efficiency) combine to produce two systems with nearly equal sensitivities, expressed in terms of CCD counts-per-x-ray incident for a given scintillator thickness. However, system statistical performance is largely dominated by the gamma photon-scintillator interaction, and LSO absorbs approximately three times the number of gamma photons, per given thickness of scintillator.

This statistical difference can be seen in a lead step wedge measurement. Figure 11 shows two line-outs of a radiograph taken of a lead step wedge target. The step target includes $6 \mathrm{~mm}$ of PMMA, along with a gradation that varies in 10 steps from 0.9 to $53 \mathrm{~mm}$ of $\mathrm{Pb}$. The scintillators were each 2.2-mm thick. The data shown is the result of a 140 pixel average, along the width of a step. The original images were smoothed with a 3.0 point median filter and star removal algorithm. By observing the noise in the individual steps, one can see that the noise level for the second step in the CsI data is comparable to the noise in the eighth step of the LSO data. This is a transmittance difference of nearly 10 times.

This observed difference in SNR can be calculated for these scintillators in a SABRE spectrum and, given a dose, resolution element size and material thickness. Figure 12 shows a calculation of SNR for a target dose of 3 rads at a first conjugate distance of 1 meter. For this calculation, in addition to the lead target, the beam was attenuated by $20 \mathrm{~mm}$ of $\mathrm{Al}$ and a composite of wood and $\mathrm{Cu}$ making up the camera RF enclosure. This calculation shows an expected $\sim 2 \mathrm{x}$ improvement in SNR when comparing LSO to CsI. As shown in Figure 12, this SNR difference tracks over a range of Pb thickness in the step wedge. When corrected for the dose differences in these two shots, and the square-root dependence of SNR on dose, the data is in rough agreement with calculation.

$$
\sqrt{10 * \frac{1.6 \mathrm{rads}}{2.3 \mathrm{rads}}}=2.6 \approx 2
$$

Large differences also existed in the quality of the surface finish. LSO is a hard material that can be polished to an optical finish. CsI is very soft. While the vendor did diamond-turn the CsI samples, they were scratched during shipment. Most of the imperfections were removed with flat field correction, but residuals from the scratches may account for the excessively (2.6 versus 2.0 ) degraded SNR. 


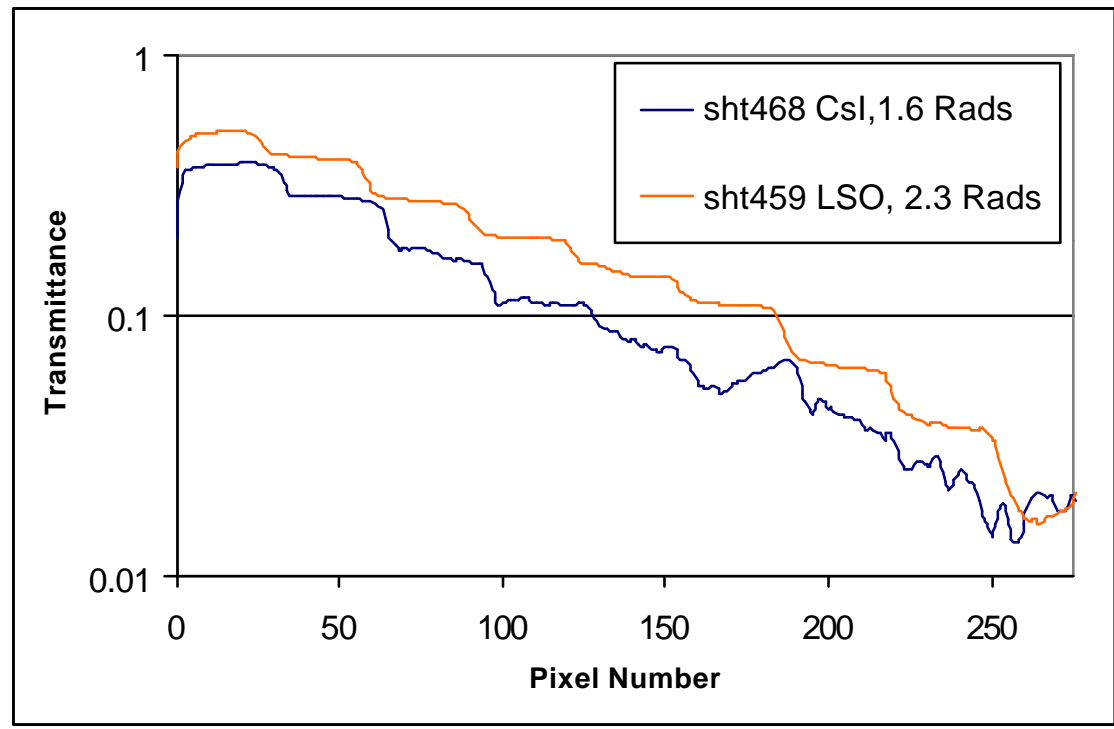

Figure 11. Comparison of lead stepwedge data with LSO and CsI scintillators.

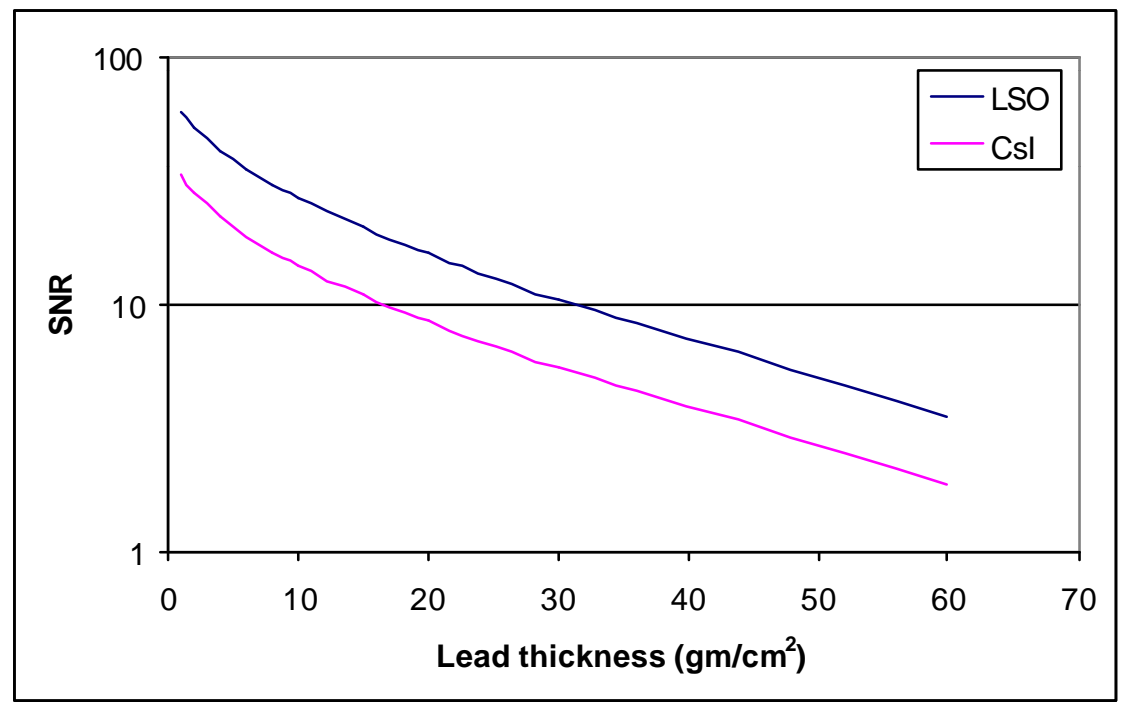

Figure 12. SNR calculation for LSO and CsI with dose of 3 rads @ 1 meter.

\subsection{DISCUSSION}

Scintillator thickness is clearly an important parameter in the optimization of a radiographic system. For this particular detection system, an LSO thickness on the order of $3 \mathrm{~mm}$ will provide optimized performance in the scintillator-plane frequency range of 0.5 to $2.5 \mathrm{~mm}^{-1}$, when used with this detection system. These measurements are clearly system specific. The system resolution (MTF) is described by convolution of the x-ray spot, scintillator-plane scatter and optical-depth-of-field. This MTF is then 
combined with the statistical performance of the scintillator. Statistical performance is determined by $\mathrm{x}$ ray source spectra, scintillator composition and scintillator conversion efficiency. Calculations show that with these systems the dominant term in statistical performance is $\mathrm{x}$-ray conversion efficiency.

This paper presented a three-dimensional parameter study. DQE is presented as a function of spatial frequency and scintillator thickness for a specific, optically fast, detection system and x-ray source configuration. Separation of the MTF into separate components of source spot, optical blur, and radiation blur would allow optimization of all three components in a multi-dimensional space.

\subsection{ACKNOWLEDGMENT}

I would like to thank my collaborators Doug Fulton (LANL DX-3) and Jim Gatling (BN) for programmatic support; Mike D. Ulibarri (LANL DX-3) for design, construction and use of the detection system at SABRE; Scott Watson (LANL DX-3) and Nick King (LANL P-23) for numerous technical discussions and advice. I also thank Peter Menge and the SNL SABRE crew for outstanding support over the several months it took to obtain these and other measurements; Debra Archuleta (LANL DX-3), Steven Jaramillo (LANL P-23) and Peter Pazuchanics (LANL P-23) for computer, data acquisition, and mechanical support.

\subsection{REFERENCES}

1. P.R. Menge, D.L. Johnson, J.E. Maenchen, D.C. Rovang, B.V. Oliver, D.V. Rose, and D.R. Welch, "Rod Pinch Radiography Source Optimization at 2.3 MV," to be published in Proceedings of the 13th IEEE International Pulsed Power Conference, edited by R.E. Reinovsky and M.A. Newton, Las Vegas, Nevada, June 17-22, 2001.

2. DARHT Technical note 118, The Use of Dense, Monolithic, Scintillator Membranes as High Resolution Imaging Detector for MegaVolt Radiography and U1-A and DARHT, Scott Watson, et al., November 29, 1999.

3. Experimental Efforts and Results in Finding New Heavy Scintillators, Derenzo and Moses, LBL, Proceedings of CRYSTAL 2000m International Workshop on Heavy Scintillators for Scientific and Industrial Applications, Chamonix, France, September 22-26, 1992, pg 125-135.

4. Spectra calculated by Dr. Peter Menge of SNL using monte-carlo method described in following: J.A. Halbleib, R.P. Kensek, G.D. Valdez, S.M. Seltzer, and M.J. Berger, IEEE Trans. Nucl. Sci., 39, 1025 (1992).

5. Image Science, Dainty and Shaw, Academic Press 1974, pg 313. 


\section{DISTRIBUTION}

U.S. Department of Energy

National Nuclear Security Administration

Nevada Operations Office

Technical Information Resource Center

P.O. Box 98518

Las Vegas, NV 89193-8518

U. S. Department of Energy

Office of Scientific and Technical Information

P. O. Box 62

Oak Ridge, TN 37831-0062

U.S. Department of Energy

National Nuclear Security Administration

Nevada Operations Office

Public Reading Facility

P.O. Box 98521

Las Vegas, NV 89193-8521

NLV 040 